\title{
Cerebral Infarction Caused by a Tortuous Subclavian Artery: a Case Report
}

\author{
Ku-Eun Lee, MD', Eun Hye Jeong, MD', Hyun-Jeung Yu, MD', Soung Sil Choi, MD², \\ Jong Kook Rhim, MD³, Kye Won Kwon, MD4, Sook Young Roh, MD'
}

Tortuous arteries are common clinical observation. Although mild tortuosity is asymptomatic, severe tortuosity can lead to ischemic attack in several organs. With advances in imaging technology, an increasing number of tortuous vessels have been detected. The purpose of this report is to describe a case of acute cerebral infarction due to tortuous subclavian artery and to review the literature.

Key Words : Cerebral infarction; Arterial tortuosity; Arteries/abnormalities; Subclavian artery

Tortuous or twisted arteries and veins are commonly observed in both humans and animals. Although mild tortuosity is a common anomaly that does not cause clinical symptoms, severe tortuosity can lead to various serious symptoms. Histologically, the arteries in a patient with arterial tortuosity can show a reduced wall thickness or lumen diameter. Together, the clinical presentation and histological findings suggest that this is a connective tissue disorder. Although other connective tissue disorders have been linked to stroke [1], arterial tortuosity and ischemic stroke are not rarely associated. We report a case of ischemic stroke caused by a twisted blood vessel.

\footnotetext{
'Departments of Neurology, ${ }^{2}$ Thoracic and Cardiovascular Surgery, ${ }^{3}$ Neurosurgery, ${ }^{4}$ Pathology, Bundang Jesaeng General Hospital, Seongnam, Korea

Received October 31, 2013; accepted after revision December 30, 2013.

Correspondence to: Ku-Eun Lee, MD, Department of Neurology, Bundang Jesaeng General Hospital, 255-2 Seohyun-dong, Bundang-gu, Seongnam-si, Gyeonggi-do 463-050, Korea.

Tel. 82.31.779.0216 Fax. 82.31.779.0879

E-mail: nmgoose@naver.com

This is an Open Access article distributed under the terms of the Creative Commons Attribution Non-Commercial License (http://creativecommons.org/licenses/by-nc/3.0) which permits unrestricted non-commercial use, distribution, and reproduction in any medium, provided the original work is properly cited.
}

\section{CASE REPORT}

A 35-year-old male patient was admitted with headache, visual disturbance and left hemiparesis. He had no history of hypertension, diabetes mellitus or other cardiac disease, and he was currently a 10-packper-year smoker. The headache was described as feeling like a tight band around his head. He denied any nausea or vomiting. The neurological examination results were normal, although he complained of numbness in his left side. Upon admission, his vital signs were stable. A chest X-ray and laboratory findings were normal. Brain magnetic resonance image (MRI) revealed an acute infarction in the right lateral thalamus (Fig. 1A). Brain CT angiography indicated a severe tortuosity with elongation at the right subclavian artery and stenosis at the right posterior cerebral artery. Negative transthoracic and transesophageal echocardiogram and Holter monitoring ruled out a cardiac source of the embolus. Hypercoagulation tests for antinuclear antibody, anticardiolipin, antithrombin III, protein C, protein $S$ and lupus anticoagulant were all negative.

Digital subtraction angiography demonstrated a severe tortuosity with elongation of the right subclavian artery (Fig. 1B). The carotid vessels on both sides were normal. On hospital day 4, he underwent a right subclavian exposure through a supraclavicular approach. The 
wall of the proximal right subclavian artery was tortuous and enlarged. The abnormal right subclavian artery was resected after clamping the diseased portion at both ends. The proximal right subclavian artery was then anastomosed directly at the right common carotid artery directly. After an end to end anastomosis, magnetic resonance angiography (MRA) revealed recanalization of the subclavian artery (Fig. 1C). The histopathological findings revealed tortuous lumen and myxoid degeneration of the wall without evidence of vasculitis (Fig. 1D).

\section{DISCUSSION}

In this case, the findings of cerebral infarction and striking tortuosity of the manor arteries were observed. We searched for other stroke risk factors such as coagulopathy or, connective tissue disease, but the patient did have any other risk factors. Because the extensive search for other risk factors was negative, arterial tortuosity is likely to have played a key role.

Tortuous blood vessels have become a common angiographic finding in many studies and clinical screenings. Various forms have been reported in clinical investigations, and some of the common
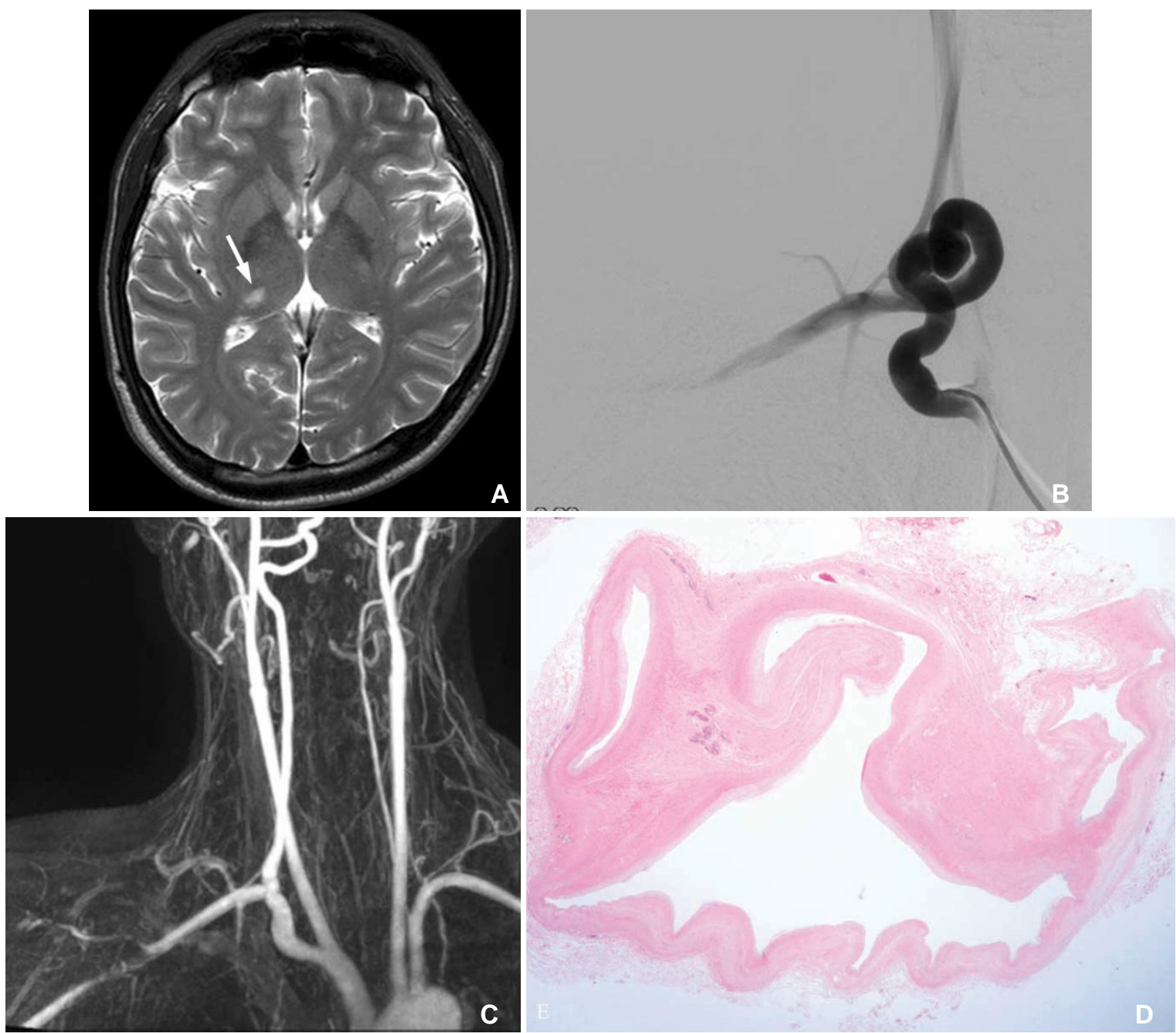

Fig. 1. A. T2-weighted MRI shows an acute infarction in the right thalamus (arrow). B. Catheter angiography reveals a tortuous right subclavian artery. C. On MRA of the neck, the subclavian artery is straightened after an end to end anastomosis. D. The specimen of subclavian artery shows irregular tortuous lumen in $\mathrm{H} \& \mathrm{E}$ stain $(\times 10)$. 
include curving/curling, angulations, twisting, looping and kinking [2]. Blood vessel tortuosity is a widely observed vascular anomaly that affects a range of vessels. Tortuosity has been reported in the aorta and capillaries, as well as in the vertebral, subclavian, coronary, cerebral and internal carotid arteries [3]. Clinical and experimental studies have demonstrated a strong association between vessel tortuosity and the mechanical characteristics of the vessel, such as blood pressure, blood flow, axial tension and the wall structure.

Tortuosity of the cerebral arteries was found to be associated with the severity of hypertension $[4,5]$. In addition, arterial tortuosity has been associated with reduced axial tension or elongation of the arteries. A certain level of axial tension is essential to maintain the stability of the arteries and prevent tortuosity. Axial tension may decrease with excessive growth of the arteries and the individual age [6].

Weakening of the arterial wall has been associated with tortuous vessels. The degradation of elastin, an important extracellular matrix component, weakens the arterial wall and leads to tortuosity. Evidence strongly suggests that high blood pressure, reduced axial tension and weakened wall stiffness may also play important roles [7].

Tortuosity increases the resistance to blood flow and severe tortuosity can decrease or even occlude blood flow. Although arterial tortuosity and stroke are not commonly associated, autopsy results have suggested that infarction of other organs may be a consequence of tortuosity. Clinically significant stenosis in the cerebral vasculature are well described feature of the arterial tortuosity syndrome [8]. Although arterial tortuosity to be a stroke risk factor is controversial, severe tortuous or twisting arteries have been treated by surgical correction and found to lower the risk of stroke. Benes and Netuka reported two cases of posterior fossa ischemia [3]. Medical therapy failed in both cases. Other source of embolization, except middle part of vertebral artery kinking were excluded. A simple surgical technique was employed. Symptom of cerebral ischemia in the presence of large vessel tortuosity may be caused either by embolization. One possibility that a disruption of the elastic fibers and a fragmentation of the internal elastic membrane altered the endothelial surface and lead to arterial thrombosis and embolization of the posterior cerebral artery. From clinical, as well as diagnostic investigations, we cannot fully determine if the subclavian artery tortuosity was the source of embolization. Nevertheless, all other source of were excluded. The main reason to offer the surgery was the prevention of recurrent stroke in young patient. We report an association between arterial tortuosity and ischemic stroke. Although the pathogenesis of the stroke is uncertain and stroke is rare occurrence, surgical treatment should be carefully considered.

\section{References}

1. North KN, Whiteman DA, Pepin MG, Byers PH. Cerebrovascular complications in Ehlers-Danlos syndrome type IV. Ann Neurol 1995;38:960-964

2. Pancera P, Ribul M, Presciuttini B, Lechi A. Prevalence of carotid artery kinking in 590 consecutive subjects evaluated by Echocolordoppler. Is there a correlation with arterial hypertension? J Intern Med 2000;248:7-12

3. Benes V, Netuka D. Surgical correction of symptomatic vertebral artery kinking. Br J Neurosurg 2003;17:174-178

4. Hiroki M, Miyashita K, Oda M. Tortuosity of the white matter medullary arterioles is related to the severity of hypertension. Cerebrovasc Dis 2002;13:242-250

5. Callewaert BL, Willaert A, Kerstjens-Frederikse WS, De Backer J, Devriendt K, Albrecht B, et al. Arterial tortuosity syndrome: clinical and molecular findings in 12 newly identified families. Hum Mutat 2008;29:150-158

6. Sugawara J, Hayashi K, Yokoi T, Tanaka H. Age-associated elongation of the ascending aorta in adults. JACC Cardiovasc Imaging 2008;1:739-748

7. Han HC. Twisted blood vessels: symptoms, etiology and biomechanical mechanisms. J Vasc Res 2012;49:185-197

8. Cartwright MS, Hickling WH, Roach ES. Ischemic stroke in an adolescent with arterial tortuosity syndrome. Neurology 2006;67:360-361 